\title{
Clinical effect of "工”-shape partial colpocleisis in elderly women with pelvic organ prolapse: a retrospective cohort study
}

\author{
Hongtao $\mathrm{Lv}^{1}$, Yingrui $\mathrm{Fu}^{2}$, Ting Zhou ${ }^{1}$, Yingying Cui ${ }^{1}$, and Fengnian Rong${ }^{2}$ \\ ${ }^{1}$ Shandong Qianfoshan Hospital \\ ${ }^{2}$ Affiliation not available
}

September 9, 2020

\begin{abstract}
Objective This study was performed to determine the effect of "工”-shape partial colpocleisis (modified LeFort partial colpocleisis) in elderly women with advanced pelvic organ prolapse. Design A retrospective cohort study. Setting One public hospital in China. Population 67 women with grade 3 or 4 POP undergoing surgery from January 2015 to December 2019. Methods This retrospective cohort study involved women who underwent "工"-shape partial colpocleisis for advanced pelvic organ prolapse. We collected data regarding the patients' baseline characteristics, comorbidities, severity of prolapse, operation time, and postoperative complications. Subjective postoperative outcomes and patient satisfaction levels were also assessed. Main Outcome Measures Operation time, intraoperative blood loss, postoperative hospital stay, operative complications and patients' satisfaction. Results Overall, 67 women were included. The mean operation time was $71.3 \pm 21.7$ minutes, and the mean intraoperative blood loss was $81.3 \pm 36.7 \mathrm{ml}$. The mean postoperative hospital stay was $4.6 \pm 3.0$ days. No intraoperative complications occurred, and $8.96 \%$ of patients had postoperative complications. All patients were satisfied with the outcome of the "工"-shape partial colpocleisis procedure. Conclusion The "工"-shape partial colpocleisis procedure had a high satisfaction rate and a positive impact on pelvic symptoms. It was associated with minimal adverse events in women with advanced apical pelvic organ prolapse. This procedure should be considered a treatment option for advanced apical pelvic organ prolapse in selected elderly women who do not wish to conserve their vaginal coital function.
\end{abstract}

\section{Hosted file}

Clinical effect of "\begin\{CJK\}\{UTF8\}\{gbsn\} \end\{CJK\}\selectlanguage\{english\}"-\selectlanguage\{english\}s. } available at https://authorea.com/users/357701/articles/480139-clinical-effect-of-\%E5\%B7\%A5shape-partial-colpocleisis-in-elderly-women-with-pelvic-organ-prolapse-a-retrospectivecohort-study 